\begin{tabular}{|c|l|}
\hline Title & Modeling and Characterization of Cloud Dynamics \\
\hline Author(s) & Y anagita, Tatsuo; Kaneko, Kunihiko \\
\hline Citation & $\begin{array}{l}\text { PHY SICAL REVIEW LETTERS, 78(22), 4297-4300 } \\
\text { https://doi.org/10.1103/PhysRevLett.78.4297 }\end{array}$ \\
\hline Issue Date & 1997-06-02 \\
\hline Doc URL & http://hdl.handle.net/2115/5755 \\
\hline Rights & Copyright $\odot$ 1997 A merican Physical Society \\
\hline Type & article \\
\hline File Information & PRL78-22.pdf \\
\hline
\end{tabular}

Instructions for use 


\title{
Modeling and Characterization of Cloud Dynamics
}

\author{
Tatsuo Yanagita* \\ Research Institute for Electronic Science, Hokkaido University, Sapporo, Hokkaido 060, Japan
}

Kunihiko Kaneko

Department of Pure and Applied Sciences, University of Tokyo, Komaba, Meguro-ku, Tokyo 153, Japan

(Received 8 July 1996; revised manuscript received 3 March 1997)

\begin{abstract}
A phenomenological model for cloud dynamics is proposed, which consists of the successive operations of the physical processes: buoyancy, diffusion, viscosity, adiabatic expansion, fall of a droplet by gravity, descent flow dragged by the falling droplet, and advection. Through extensive simulations, the phases corresponding to stratus, cumulus, stratocumulus, and cumulonimbus are found by changing the ground temperature and the moisture of the air. They are characterized by order parameters such as the cluster number, perimeter-to-area ratio of a cloud, and Kolmogorov-Sinai entropy. Though our model is simple and constructive, it may shed some light on the true nature of clouds. [S0031-9007(97)03289-4]
\end{abstract}

PACS numbers: 92.60.Jq, 47.52.+j, 92.40.Cy

Cloud dynamics plays an important role in the climate system, weather forecast, geophysics, and so on. However this elementary process in meteorology is very much complicated because it consists of different time and space scales and the phase transition from condensed phase to gas is coupled with the motion of atmosphere. Even if the flow of the atmosphere were known with accuracy using the Navier-Stokes equation, we could not discuss the morphology of clouds. In order to investigate such a complex system, construction of a phenomenological model is essential. In this Letter, we introduce a model of cloud formation which reproduces the diversity of cloud patterns. Characterizations of four cloud phases are also given.

Coupled map lattices (CML) [1] or cell dynamics (CD) [2] are useful to study the dynamics of spatially extended systems [3]. Recently, the methods have successfully been applied to spinodal decomposition [2], RayleighBénard convection [4], the boiling transition [5], and so on [6].

Here we construct a CML (or CD) model of a cloud in a two-dimensional space. The modeling is based on the separation and successive operation of procedures, which are represented as maps acting on a field variable on a lattice [3]. Here, we choose a two-dimensional square lattice $(x, y)$ with $y$ as a perpendicular direction, and assign the velocity field $\vec{v}^{t}(x, y)$, the mass of the vapor $w_{v}^{t}(x, y)$, the condensed phase $w_{\ell}^{t}(x, y)$, and the internal energy $E^{t}(x, y)$ as field variables at time $t$. Since the latent heat between solid and liquid is much smaller than that between condensed and gas phases, the distinction of liquid and solid is neglected in the present Letter, and we often use the term "liquid" to represent the condensed phase. The dynamics of these field variables consists of Lagrangian and Eulerian parts. For the latter part, we adopt the following processes (here we consider spatial scale such that the centrifugal and Coriolis forces are ne- glected): (1) heat diffusion; (2) viscosity; (3) buoyance force; (4) the pressure term requiring $\operatorname{div} \vec{v}$ to be 0 , in an incompressible fluid (if the vertical air motion is confined within a shallow layer, the motion of atmosphere can be regarded as incompressible flow [7]). Here, we use the discrete version of $\operatorname{grad}(\operatorname{div} \vec{v})$ which refrains from the growth of $\operatorname{div} \vec{v}$. Indeed, we have already constructed the CML representations of the above four procedures [4] that agree with experiments on the Rayleigh-Bénard convection, which is a cardinal role for cloud dynamics; (5) diffusion of vapor $w_{v}^{t}(x, y)$; (6) adiabatic expansion; assuming the adiabatic process and the equilibrium ideal gas with gravity field, we adopt such an approximation that the temperature of the parcel risen from the height $y$ to $y+\Delta y$ is decreased in proportion to the displacement $\Delta y$. Thus the temperature $E^{t}(x, y)$ is decreased in proportion to $v_{y}^{t}(x, y)$; (7) phase transition from vapor $w_{v}^{t}(x, y)$ to liquid $w_{\ell}^{t}(x, y)$ and vice versa accompanied by the latent heat. Here, we use the simplest type of the bulk watercontinuity model in meteorology [8]. The dynamics is represented as a relaxation to an equilibrium point $w^{*}(x, y)$ which is a function of temperature $E^{t}(x, y) ;(8)$ the dragging force; assuming that the droplets are uniform in size and fall with the terminal velocity $V$ with neglect of the relaxation time to it, the dragging force is proportional to the product of the relative velocity $\left[v_{y}^{t}(x, y)-V\right]$ and the density of droplets $w_{\ell}^{t}(x, y)$ at a lattice site.

Combining these dynamics, the Eulerian part is written as the successive operations of the following mappings [hereafter we use the notation for discrete Laplacian operator: $\quad \Delta A(x, y)=\frac{1}{4}\{A(x-1, y)+A(x+1, y)+$ $A(x, y-1)+A(x, y+1)-4 A(x, y)\}$ for any field variable $A(x, y)$ ]: For convenience, we represent state variables after an operation of each procedure with the superscript $t+1 / n$ where $n$ is the total number of procedures. 
Buoyancy and dragging force:

$$
v_{y}^{t+1 / 3}(x, y)=v_{y}^{t}(x, y)+\frac{c}{2}\left[E^{t}(x+1, y)+E^{t}(x-1, y)-2 E^{t}(x, y)\right]-\gamma w_{\ell}(x, y)\left[v_{y}(x, y)-V\right] .
$$

Viscosity and pressure effect:

$$
\begin{aligned}
\vec{v}^{t+2 / 3}(x, y)= & \vec{v}^{t+1 / 3}(x, y)+\nu \Delta \vec{v}^{t+1 / 3}(x, y) \\
& +\eta \operatorname{grad}\left[\operatorname{div} \vec{v}^{t+1 / 3}(x, y)\right],
\end{aligned}
$$

with $\operatorname{grad}(\operatorname{div} \vec{v})$ as its discrete representation on the lattice [4].

Thermal diffusion and adiabatic expansion:

$E^{t+1 / 3}(x, y)=E^{t}(x, y)+\lambda \Delta E^{t}(x, y)-\beta v_{y}^{t}(x, y)$.

Diffusion of vapor:

$$
w_{v}^{t+1 / 3}(x, y)=w_{v}^{t}(x, y)+\lambda \Delta w_{v}^{t}(x, y) .
$$

Phase transition: To get the procedure, we use the discretized version of the following linear equations for the relaxation to equilibrium point $w^{*}$

$$
\begin{aligned}
d w_{v}(x, y) / d t & =+\alpha\left[w_{v}(x, y)-w^{*}\right], \\
d w_{\ell}(x, y) / d t & =-\alpha\left[w_{v}(x, y)-w^{*}\right] \\
d E(x, y) / d t & =-Q\left[d w_{v}(x, y) / d t-d w_{\ell}(x, y) / d t\right],
\end{aligned}
$$

$$
w^{*}= \begin{cases}A \exp [q /(E+\text { const })], & \text { if }>W(x, y), \\ W(x, y), & \text { otherwise, }\end{cases}
$$

which form is chosen to be consistent with the ClausiusClapeyron's equation $\exp (-q / T)$ [9], while $W(x, y)=$ $w_{\ell}(x, y)+w_{v}(x, y)$ is the total mass of water.

The Lagrangian scheme expresses the advection of velocity, temperature, liquid, and vapor. This process is expressed by the motion of a quasiparticle on each lattice site $(x, y)$ with velocity $\vec{v}(x, y)$. We adopt the method presented in [4], while for the liquid variable $w_{\ell}(x, y)$, we also include the fall of a droplet with a final speed $V$. Thus, the quasiparticle moves to $(x+$ $\left.v_{x}(x, y), y+v_{y}(x, y)-V\right)$ to allocate $w_{\ell}(x, y)$ at its neighbors. Through this Lagrangian procedure, the energy and momentum are conserved.

Summing up, our dynamics is given by successive applications of the following step:

$$
\begin{aligned}
& \left\{\begin{array}{c}
\vec{v}^{t}(x, y) \\
E^{t}(x, y) \\
w^{t}(x, y)
\end{array}\right\} \stackrel{\text { Buoyancy+Dragging }}{\rightarrow}\left\{\begin{array}{c}
\overrightarrow{\boldsymbol{v}}^{t+1 / 3}(x, y) \\
E^{t}(x, y) \\
w^{t}(x, y)
\end{array}\right\} \stackrel{\text { Viscosity+Pressure }}{\rightarrow}\left\{\begin{array}{c}
\vec{v}^{t+2 / 3}(x, y) \\
E^{t}(x, y) \\
w^{t}(x, y)
\end{array}\right\} \\
& \stackrel{\text { Diffusion }}{\longrightarrow}\left\{\begin{array}{l}
\vec{v}^{t+2 / 3}(x, y) \\
E^{t+1 / 3}(x, y) \\
w^{t+1 / 3}(x, y)
\end{array}\right\} \text { Phase transition }\left\{\begin{array}{l}
\vec{v}^{t+2 / 3}(x, y) \\
E^{t+2 / 3}(x, y) \\
w^{t+2 / 3}(x, y)
\end{array}\right\} \stackrel{\text { Advection+Gravity }}{\rightarrow}\left\{\begin{array}{l}
\vec{v}^{t+1}(x, y) \\
E^{t+1}(x, y) \\
w^{t+1}(x, y)
\end{array}\right\} \text {. }
\end{aligned}
$$

For the boundary, we choose the following conditions. (1) Bottom plates: assuming the correspondence between $E$ and the temperature, we choose $E(x, 0)=E_{0}$. (2) Top plates: we choose the no-flux condition $E_{t}\left(x, N_{y}\right)-$ $E_{t}\left(x, N_{y}-1\right)=0$. For both the plates, we choose the slip condition for the velocity field, and adopt the reflection boundary for the Lagrangian procedure. The liquid and vapor are fixed at zero for both the plates. (3) Side walls at $x=0$ and $x=N_{x}$ : we use periodic boundary conditions.

The basic parameters in our model are the temperature $E_{0}$ at the ground, the Prandtl number (ratio of viscosity to heat diffusion $\nu / \lambda$ ), adiabatic expansion rate $\beta$, the terminal velocity of liquid droplets $V$, the coefficient for the dragging force $\gamma$, the phase transition rate $\alpha$, the latent heat $Q$, and the aspect ratio $\left(N_{x} / N_{y}\right)$. Hereafter we fix these parameters as $\lambda=0.2, \eta=\nu=0.2, c=$ $0.2, \beta=0.2, V=0.2, \gamma=0.2, \alpha=0.2, Q=0.2$, and study the change of the morphology in the cloud as the ground temperature $E_{0}$ and the total mass of water $W=\sum_{x, y}\left[w_{v}(x, y)+w_{\ell}(x, y)\right]$ are varied (note that $W$ is conserved). Our result is rather robust against the changes of parameters. The convection patterns are qualitatively the same over the changes of the parameters $\lambda, \eta, \mu, c$ from 0.1 to 0.4 [4], while the morphology of clouds is not affected over the regions $\gamma=V=0.05 \sim 0.5$ and $\beta=0.15 \sim 0.35$.
To see the spatiotemporal dynamics, the evolution of the mass of the liquid $w_{\ell}(x, y)$ is studied. In Fig. 1, four typical time evolutions of $w_{\ell}(x, y)$ are plotted. By changing $E_{0}$ and $W$, the following four types of clouds have been found: (a) "stratus," (b) "cumulus," (c) "cumulonimbus" $(\mathrm{Cb})$, and (d) "stratocumulus" $(\mathrm{Sc})$.

Stratus is a thin layered pattern of cloud, while cumulus is a thick lump of cloud. These two patterns are rather stable, while the other two patterns are dynamically unstable. At Sc, a thin layered cloud pattern is torn into pieces and small fragments of clouds are scattered. These scattered clouds vanish while a new layered cloud is formed again later. The formation and annihilation of clouds are periodically repeated.

There is some difference in the static pattern of (c) and that of cumulus. It is thicker in height than cumulus. We have found a marked dynamical difference behind this difference. The flow pattern of (c) is turbulent in contrast to (b). The cloud pattern of (c) is unstable. The clouds split and coalesce repeatedly. Therefore, we may conclude that phase (c) should correspond to the $\mathrm{Cb}$, although the cloud pattern is only suggestive.

The flow and cloud patterns are correlated. Stratus is maintained by a weak ascending current uniformly existing over all the space, while localized laminar ascending flow due to the instability of a uniform flow sustains the 


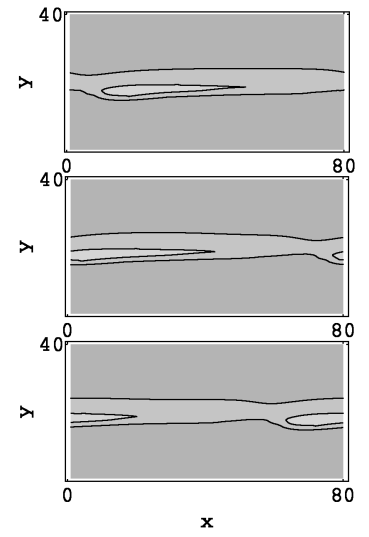

(a)

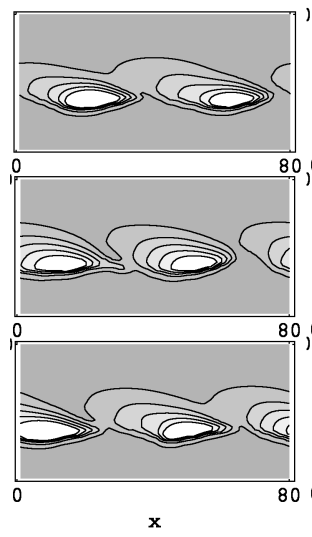

(b)

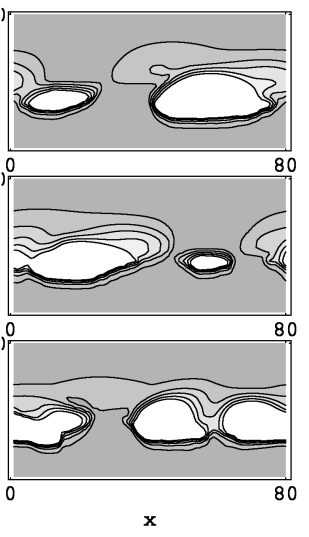

(c)

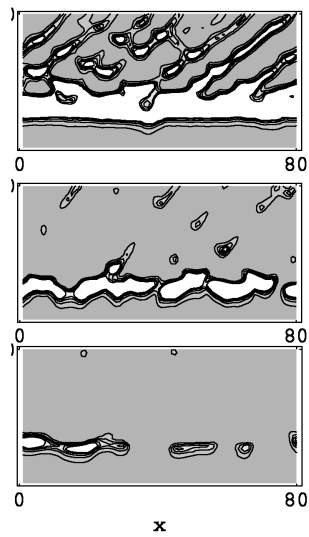

(d)

FIG. 1. Temporal evolution of cloud patterns. Snapshot of the mass of the liquid $w_{l}^{t}(x, y)$ is shown with the use of gray scale. The darker the pixel, the lower the liquid is. In other words, the white region corresponds to the high density of liquid, i.e., to a cloud region. Snapshot patterns are plotted from top to down per 200 steps after the initial 5000 steps of transients, starting from a random initial condition. (a) Stratus $\left(E_{0}=3.0, W=0.006\right)$, (b) cumulus $\left(E_{0}=3.0, W=0.007\right)$, (c) cumulonimbus $\left(E_{0}=4.0, W=0.009\right)$, (d) stratocumulus $\left(E_{0}=5.0, W=0.009\right)$. The lattice size is $\left(N_{x}, N_{y}\right)=80 \times 40$.

cumulus. As the flow gets turbulent, the cloud pattern is destabilized, leading to $\mathrm{Cb}$. On the other hand, as the ascending flow is stronger than for stratus, the cloud is thicker, whose upper part is destabilized, and the consequent turbulent flow leads to Sc.

The classification into four types is based on the comparison between our spatiotemporal pattern and the definition by meteorology [8], while the phase diagram is given in Fig. 2, which is obtained from the pattern and quantifiers to be discussed. Summarizing the diagram, a cumulus or $\mathrm{Cb}$ is observed under the condition of rich moist air while a stratus appears in small $W$ and under low temperature.

To classify these patterns quantitatively, we have measured several order parameters. First, we define a cloud cluster as connected lattice sites in which $w_{\ell}(x, y)$ is larger than a given threshold $w_{c}$. (The phase diagram of the morphology of the cloud does not depend on

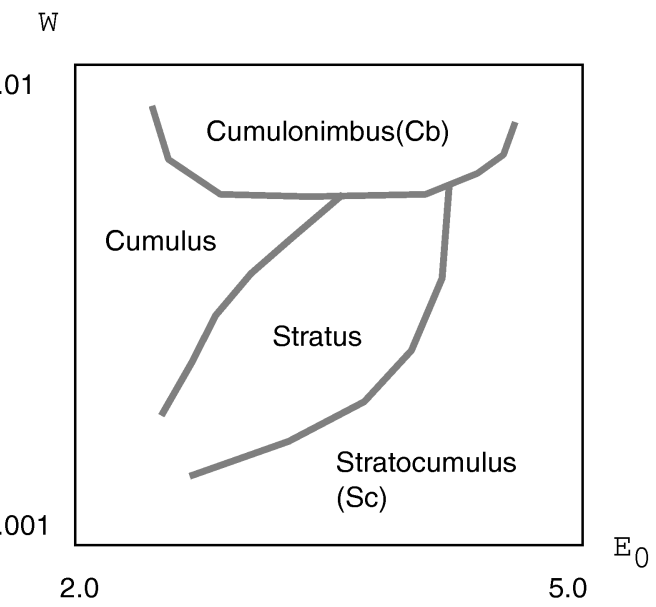

FIG. 2. Phase diagram for the morphology of the cloud. The term stratus, cumulus, cumulonimbus $(\mathrm{Cb})$, and stratocumulus (Sc) correspond to the patterns in Figs. 1(a), 1(b), 1(c), and 1(d), respectively. the choice of the threshold $w_{c}$ if $0.02<w_{c}<0.04$.) $C(t)$ is defined as the number of clusters disconnected with each other. Then, the "cloudiness" is measured by the total number of cloud sites, that is, $S(t)=$ $\sum_{x=1}^{N_{x}} \sum_{y=1}^{N_{y}} \Theta\left(w_{\ell}(x, y)-w_{c}\right)$, where $\Theta(x)$ is Heaviside function. The (temporal) average of the cluster number is large at the onset of cloud formation (i.e., small $E_{0}$ and $W)$, and at the Sc. The quantity $\langle S\rangle /\langle C\rangle$ measures the average size of each cloud cluster. It is larger at cumulus and is largest at $\mathrm{Cb}$ [Fig. 3(a)].

To characterize the difference between stratus and cumulus, the morphology of clouds should be taken into account. Roughly speaking, the stratus is a one-dimensional-like pattern while the cumulus is a twodimensional one. To see the morphological difference, we have measured the total perimeter of the cloud $L(t)=$ $\sum_{x=1, y=1}^{N_{x}, N_{y}} \sum_{\delta x= \pm 1, \delta y= \pm 1} \Theta\left(w_{\ell}(x, y)-w_{c}\right) \Theta\left(w_{c}-w_{\ell} \times\right.$ $(x+\delta x, y+\delta y))$. "Stratus order parameter" (SOP) is introduced as $\langle 1 /[S(t) / L(t) C(t)]\rangle_{t}$, the inverse of the ratio of area to perimeter per cluster. If it is large the pattern is close to a one-dimensional object. Change of SOP with $E_{0}$ and $W$ is plotted in Fig. 3(b). As is expected by the definition of SOP and the thin nature of the stratus cloud, it has a larger value at the stratus phase and takes a lower value at the cumulus. [Even by the classification by a snapshot pattern, the same phase diagram is obtained, since the dynamics and pattern are strongly correlated here. It should be noted, however, that the Sc consists of a sequence of patterns as in Fig.1(d).]

Of course, dynamical quantifiers are also interesting. For example, the fluctuations of the cluster size, SOP, and $S / C$ are larger at the $\mathrm{Cb}$ and $\mathrm{Sc}$ phases. To see the dynamics closely, we have also measured the time series of the spatial sum of the mass of the liquid $\overline{L(t)}=$ $\sum_{x, y} w_{\ell}^{t}(x, y)$, which corresponds to the cloudiness of the total space. The evolution of $\overline{L(t)}$ is almost stationary at stratus and cumulus, with only tiny fluctuations. The 


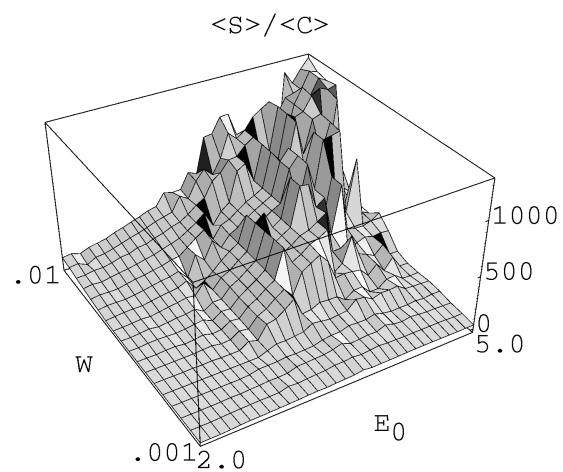

(a)

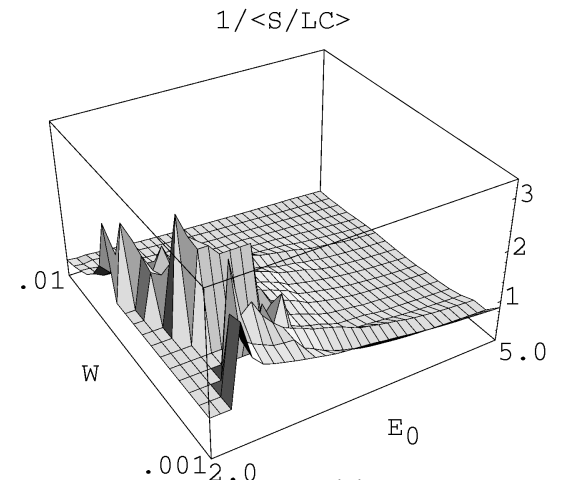

(b)

FIG. 3. (a) The average size of a cloud cluster $\langle S\rangle /\langle C\rangle$ and (b) the stratus order parameter, plotted with the change of $W$ and $E_{0}$. The average is taken over 20000 time steps after discarding 10000 steps of transients, starting from a random initial condition.

change is periodic at $\mathrm{Sc}$ and chaotic at $\mathrm{Cb}$. It should be noted that the low-dimensional collective dynamics of the total liquid emerges even if the spatiotemporal dynamics is high-dimensional chaos.

To characterize chaotic dynamics, Kolmogorov-Sinai (KS) entropy is estimated by the sum of positive Lyapunov exponents, as is plotted in Fig. 4(b). It is a larger value at $\mathrm{Sc}$ and $\mathrm{Cb}$, implying that the cloud dynamics there is spatiotemporal chaos. It is also positive at a lower temperature that corresponds to the onset of cloud formation, where the dynamics is unstable.

In summary, we have proposed a model for pattern formation of clouds by introducing a simple phase transition dynamics from liquid to vapor, so called the bulk watercontinuity model [8]. Our model reproduces the diversity of cloud patterns: stratus, $\mathrm{Sc}$, cumulus, and $\mathrm{Cb}$. This agreement implies that the qualitative feature of cloud dynamics is independent of microscopic details such as detailed droplet formation dynamics.

In order to globally understand the phenomenology, the present computationally efficient model is powerful,

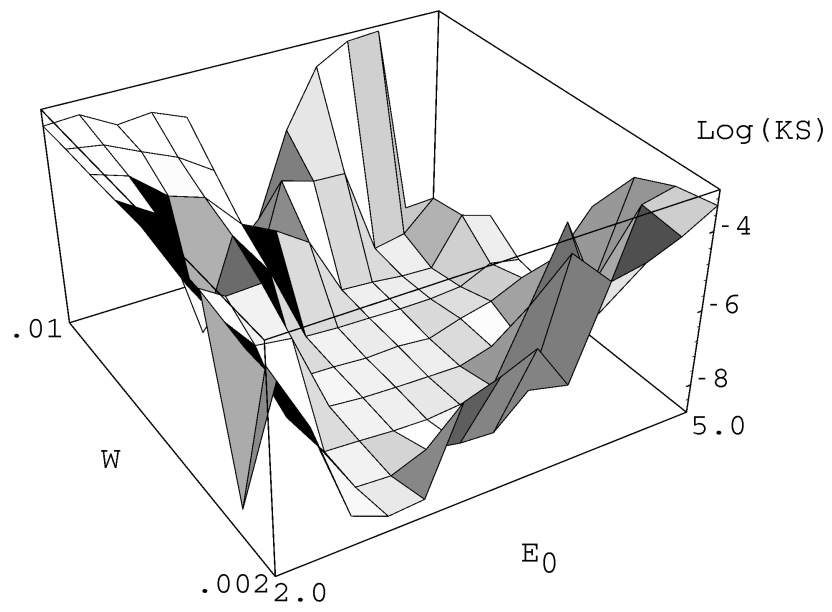

FIG. 4. KS entropy calculated by the sum of positive Lyapunov exponents is plotted versus $W$ and $E_{0}$. The first 20 Lyapunov exponents are computed by averaging over 20000 time steps after discarding the initial 5000 steps. which makes it possible for us to characterize the cloud phases. We believe that the observed phase diagram for the morphology of the cloud is valid for the cloud in nature. It is also interesting to propose that similar phase changes may be seen generally in convective dynamics including phase transitions, since detailed processes specific only to clouds are abstracted in our model. Indeed the mantle convection may provide an example of such a "generalized cloud," since perovskite shows the phase transformation from orthorhombic to tetragonal in the course of the convection. On the other hand, it may be interesting to consider a laboratory experiment to capture the essence of cloud dynamics in the general sense.

Extension of the present model to a three-dimensional case is straightforward. Through several simulations, we have again found the morphological transitions of cloud patterns. The inclusion of the phase transition between ice and liquid is also straightforward, as well as the inclusion of the Coriolis force, which enables us to study the global atmosphere dynamics of a planet.

This work is partially supported by Grant-in-Aids for Scientific Research from the Ministry of Education, Science, and Culture of Japan, and by a cooperative research program at Institute for Statistical Mathematics.

*Electronic address: http://aurora.elsip.hokudai.ac.jp

[1] K. Kaneko, Prog. Theor. Phys. 72, 480 (1984).

[2] Y. Oono and S. Puri, Phys. Rev. Lett. 58, 836 (1987).

[3] K. Kaneko, in Formation, Dynamics and Statistics of Patterns, edited by K. Kawasaki et al. (World Scientific, Singapore, 1990), Vol. I.

[4] T. Yanagita and K. Kaneko, Physica (Amsterdam) 82D, 288 (1995).

[5] T. Yanagita, Phys. Lett. A 165, 405 (1992).

[6] Chaos Focus Issue on CML [CHAOS 2 (1992)].

[7] L. D. Landau and E. M. Lifshitz, Fluid Mechanics (Pergamon, New York, 1959), Chap. 5.

[8] R. A. Houze, Cloud Dynamics, International Geophysics Series Vol. 53 (Academic Press, New York, 1993).

[9] L.D. Landau and E. M. Lifshitz, Statistical Mechanics (Pergamon, New York, 1958), Chap. 8. 